\title{
TRAGATODO(S): EL CUENTO DEL GATO Y LOS RATONES. LA VERSIÓN DE TEODORO PRÓDROMO
}

\author{
DANIEL GUTIÉRREZ \\ Universidad de Buenos Aires. Argentina
}

Resumen: Una de las particularidades, a nivel léxico, de la Catomiomaquía reside en que el término griego utilizado para designar al gato no es el muy extendido aílouros sino káta. Como en esta obra la acción es transmitida exclusivamente desde el punto de vista de los ratones, se puede conjeturar que esta sería la perspectiva con la que el autor quiere que se identifique(n) su(s) oyente/lector(es), haciendo circular un mensaje paródico-político-crítico.-

Palabras clave. Teodoro Pródromo, Catomiomaquía, efecto de extrañamiento, káta, Lector Modelo, parodia político-crítica.

\section{TRAGATODO(S): THE STORY OF CAT AND MICE. THE VERSION OF THEODORE PRODROMOS}

\begin{abstract}
One of the peculiarities, at lexical level, of Katomyomakhia is that the Greek term for the cat is not widespread ailouros but káta. As in this work action is transmitted only from the point of view of the mice, it can be assumed that this would be the perspective with which the author wants to be identified their listener/reader(s), by circulating a parodic-political-critical message.
\end{abstract}

Keywords: Theodore Prodromos, Katomyomakhia, effect of estrangement, káta, Model Reader, political-critical parody.

Recibido: 3.12 .15 - Aceptado: 21.01-16

Correspondencia: Daniel Gutiérrez.
Email almejasapera@gmail.com
Licenciatura en Filosofía (UBA), Maestrando en Metodología de la Investigación
Científica (UNLa), estudiante avanzado de Letras (UBA), miembro investigador
del proyecto UBACyT "Hagiografía, homiliética y contexto sociocultural en
Bizancio tardoantiguo: ecdótica del corpus de Leoncio de Neápolis" (2014-2017),
dirigido por el Dr. Pablo Cavallero. Fac. de Filosofía y Letras. Puán 480,
Buenos Aires. Fono: (011) 15-56096052.


Pe la vida de Teodoro Pródromo se sabe poco y nada. Se conjetura que cursó estudios de gramática, de retórica y de filosofía alcanzando así una cierta erudición-, que se inició como poeta en torno al círculo literario presidido por la Emperatriz Irene, que se ganaba la vida como maestro o profesor, que murió siendo monje con el nombre de Hilario o Nicolás y que vivió aproximadamente entre 1110 y 1170 en Constantinopla ${ }^{1}$. Dicho período corresponde a la época en que imperó la dinastía de los Comnenos, especialmente los gobiernos de Juan II Comneno (1118-1143) y Manuel I Comneno (1143-1180). Durante el mandato de este último se terminará de afianzar el "renacimiento" de la cultura clásica en Bizancio, antes promovida por Alejo I Comneno (1081-1118)².

Habiendo sido uno de los máximos representantes de la poesía bizantina en lengua popular, sobre todo a través de sus epigramas y de sus poemas "mendicantes" (que le valieron el apodo de 'Ptokhopródromo'), Teodoro Pródromo también cultivó géneros "mayores” como la novela (siendo su Rodante y Dosicles un fiel exponente del género) y la épica paródica. Esta última está representada por la Catomiomaquía, compuesta siguiendo el modelo de la comedia y la tragedia antigua (que se evidencia en su estructuración ${ }^{3}$, en su léxico y en el uso del trímetro yámbico) y, por supuesto, el del poema paródico pseudo-homérico Batracomiomaquía.

\section{I}

La Catomiomaquía retoma el tópico tradicional y popular del innato conflicto entre el gato y el ratón ${ }^{4}$. Una de las particularidades de este 'dramátion', a nivel léxico, reside en que el término griego para designar al gato no es el

$1 C f$. Hunger (1978: 517), Kazhdan (1984: 90-101), Egea (2001: 9-12), García Romero (2003: 3), Kaplan (2007: 52).

2 El renacimiento de la cultura clásica en Bizancio comenzó en el siglo XI, impulsado por el emperador Constantino IX Monómaco (1042-1055) y especialmente por su secretario Miguel Psellos; $c f$. Maier (1982: 217-221); para una exposición detallada de este proceso de recuperación del legado clásico, véase Lemerle (1971).

3 Para un claro esquema de la estructuración "dramática" de la obra, véase Hunger (1968: 51).

4 Cf. Esopo, Aílouros kaì mýes, fab. 79, ed. Perry (1952). 


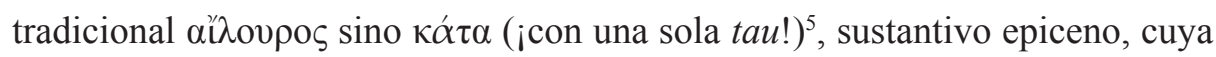
forma con doble tau es de escasa (si no nula) aparición en la literatura griega precedente ${ }^{6}$.

Observando, con respecto a estos términos, la información que brindan los diccionarios y léxicos del período clásico más reconocidos, se puede señalar que:

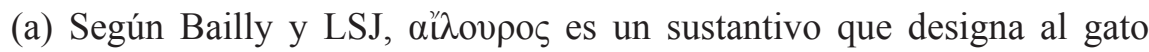
(al doméstico, "felis domesticus", según LSJ) y puede aplicarse tanto a la hembra como al macho; el término es usado, por ejemplo, en la fábula 79

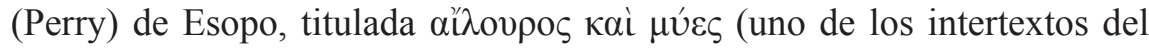

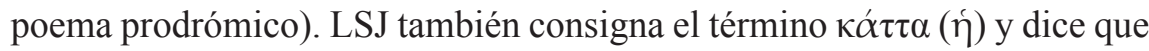

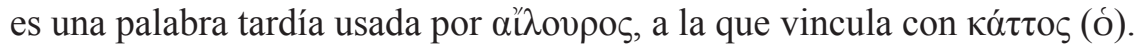

(b) Los diccionarios etimológicos de Chantraine y de Beekes no registran

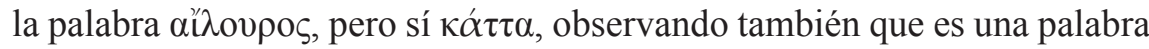

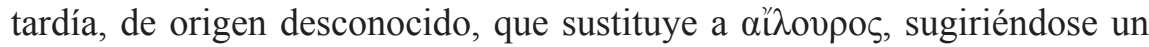
vínculo con el latín y otras lenguas europeas ( $c f$., por ejemplo, cat (ing.), Kater, Katze (alem.), kotǔka (slv.)).

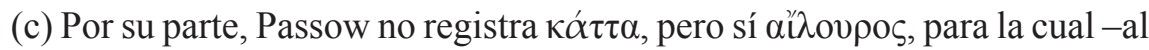

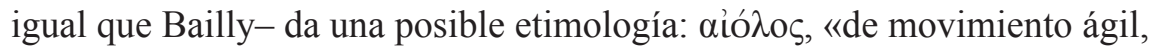
rápido», + oúpóx, «cola animal», como compuesto expresivo que designaría una característica peculiar de los gatos: el movimiento ondulante de su cola.

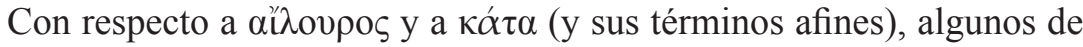
los léxicos y diccionarios sobre griego tardío, medieval y bizantino parecen ser un tanto más explícitos:

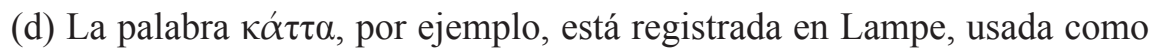
apodo o mote de alguien, aunque sin mayores apreciaciones sobre su

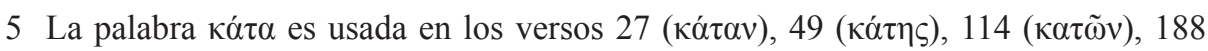

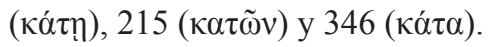

6 Se atestigua кó $\tau \tau \alpha$, por ejemplo, en un escolio a Ar. $P l .693$ para explicar el término

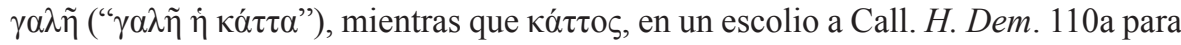

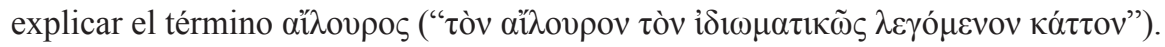


significado, excepto el testimonio de Evagrio Escolástico al que remite:

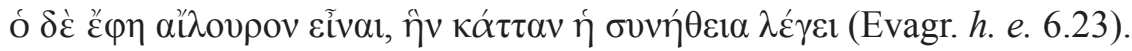

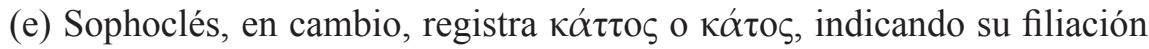
con el latín (cattus o catus, "male cat") y señalándolo como sinónimo de

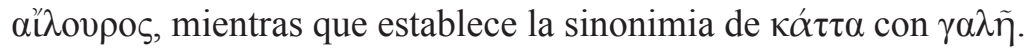

(f) Quien también registra $\kappa \alpha ́ \tau \tau \alpha$, informando que se trata de una palabra

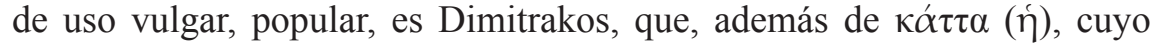
idiotismo sería $\kappa \alpha ́ \tau(\tau) \alpha$, que podría estar vinculada fonéticamente con

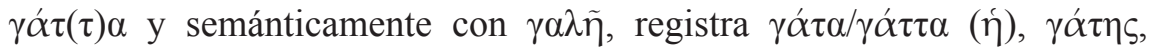

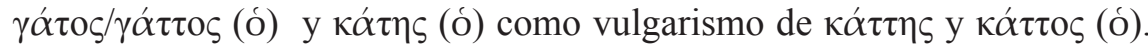

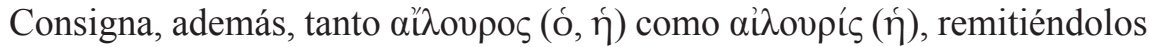
а $\gamma \alpha \lambda \tilde{\eta}$ у а $\gamma \alpha \dot{\alpha} \alpha$.

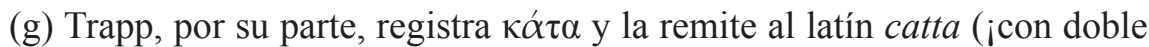

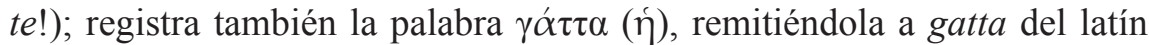

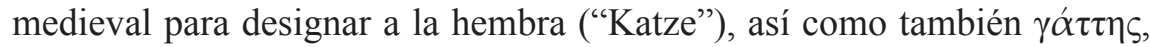

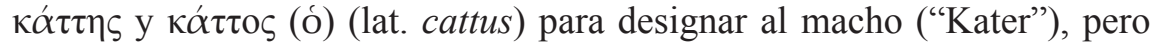

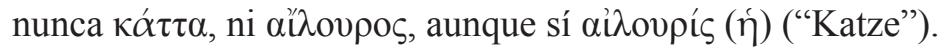

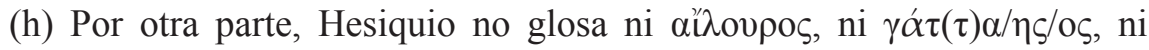

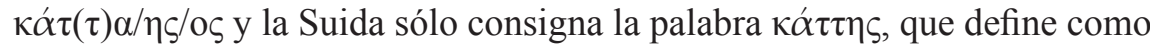

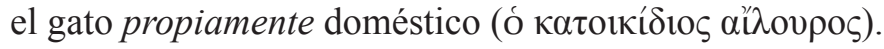

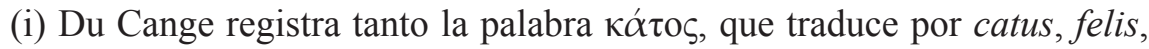
remitiendo al escolio al verso 110 del Himno a Deméter de Calímaco (ver

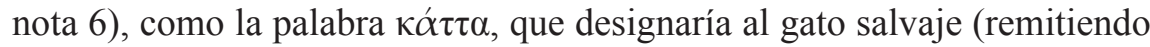
al ya mencionado testimonio de Evagr. $h$. e. 6.23) y la palabra кá $\tau \alpha$, que traduce por cata, entrada en la que remite a testimonios que la vincularían

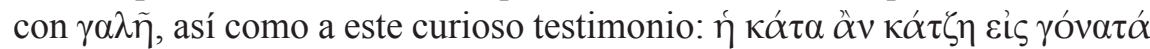
tıvos (Canonium Mathematicum adespotum), que introduce el sema de

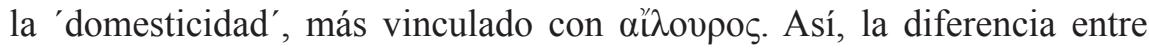

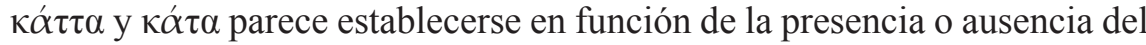

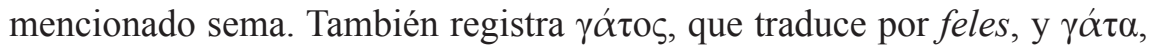
que traduce por felis. Pero a diferencia de Dimitrakos, Lampe y Trapp, quienes registran la entrada aỉ registra el término tradicional para designar al gato. 


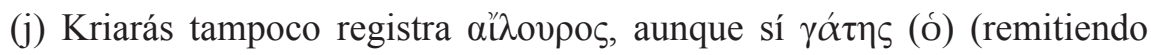

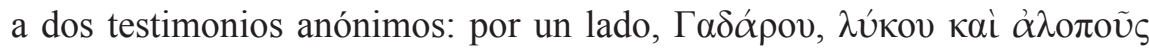

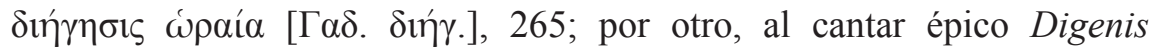

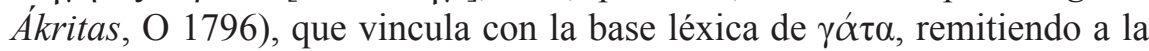

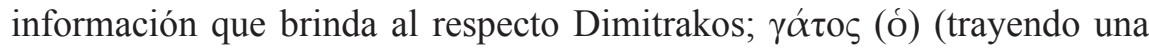
vez más el testimonio de $\Gamma \alpha \delta$. $\delta \imath \eta ́ \gamma ., 266,259)$, que refiere al macho de

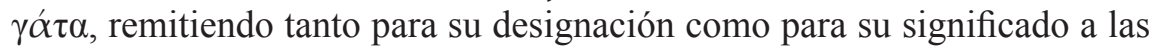
correspondientes entradas de Du Cange y de Dimitrakos e ilustrando el uso

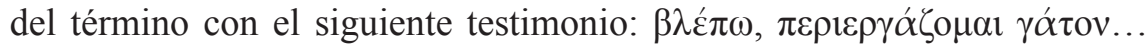

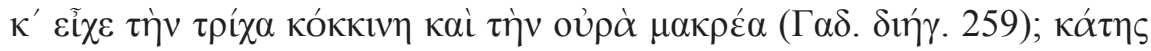

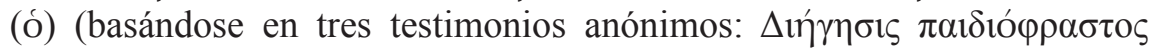
$\tau \tilde{\omega} v \tau \varepsilon \tau \rho \alpha \pi$ ó $\omega v \zeta \omega ́ \omega v, 35,55,79,125,144,184,194,564,1036,1073$;

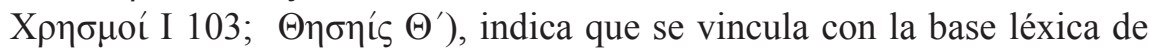
$\kappa \alpha ́ \tau \alpha$, con cambio de género, y que es una variante vulgar con significado

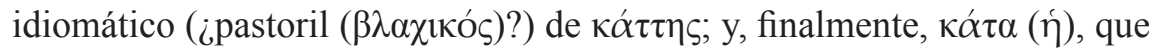
remite al latín medieval catta (¡con doble te!) (apoyándose en Niermeyer,

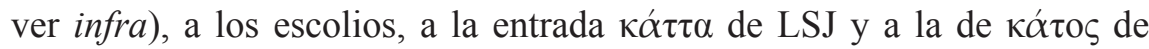
Du Cange, entendiendo su uso como una variante idiomática propia de la región del Mar Negro, utilizada para designar a los carnívoros pequeños.

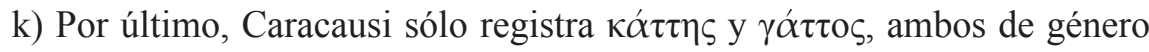
masculino; al primero lo vincula con el uso en griego tardío (a partir de

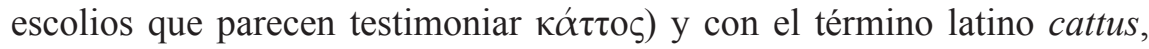
sugiriendo que podría ser una variante del griego 'romance' (neogreco)

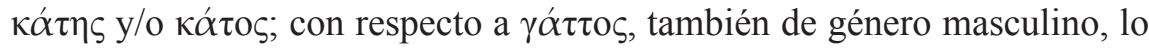
remite al latín cattus. No registra aínovpos.

En latín clásico, la palabra tradicional para designar al gato es fè lēs

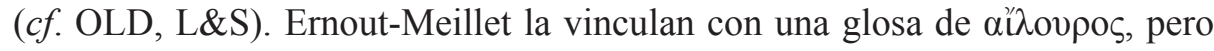
entrando en contradicción con el significado que da LSJ ("felis domesticus"), pues según estos (y también el OLD: “wild cat") designa, entre otros pequeños carnívoros, al gato salvaje. Señalan, asimismo, que el término cattus, catta (o gattus, gatta) es un término tardío que habrá de derivar en las palabras romances gatto, -a (ital.), gato, - a (esp.), chat, chatte ( $f$ r.), a la vez que observan que el uso de cattus por fèlēs correspondería a la introducción tardía del gato doméstico en $\mathrm{Roma}^{7}$; dicho término tendría una filiación con el irl. catt, con el

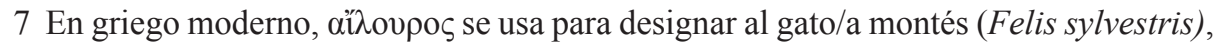


gall. cath, con el v. all. kazza, con el v. norr. kottr, con el lit. katế' y con el slv. kotûka, pudiendo provenir todos ellos de una lengua desconocida.

Niermeyer, por su parte, consigna como términos de uso latino medieval cat(t)a, cat(t)us y gat(t)us. La entrada de cata remite inmediatamente a la de catta (ambas usadas para designar a la hembra). Esta entrada a su vez remite a la de gatus. Gatus, gattus parece ser equivalente a cat(t)us (en tanto términos usados para designar al macho). Lo que se pregunta es si todos estos términos no tendrían un origen celta.

Asimismo, mientras los latinistas señalan una posible filiación con la palabra latina $m \overline{\mathrm{e}} l \overline{\mathrm{e}} s$ ("marta", "comadreja"), los helenistas/bizantinistas la emparentan con el sustantivo $\gamma \alpha \lambda \varepsilon ́ \eta$ (contr. $\gamma \alpha \lambda \tilde{\eta})$. Tal filiación se puede deber a que esta última palabra designaría probablemente a la Mustela nivalis ("marta", "comadreja"), especie más pequeña de mamífero mustélido ampliamente distribuido en Eurasia, que, domesticado, era utilizado cotidianamente en el mundo antiguo para limpiar la casa de pequeños roedores ${ }^{8}$.

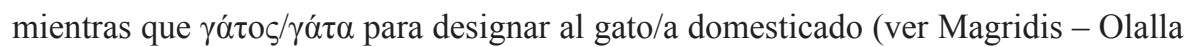
(2006: ad. loc.)).

8 Flacelière (1959) informa sobre este uso doméstico y popular de las comadrejas ya en la Atenas de Pericles. Cabe mencionar que en la editio princeps de Apostolius (ca.

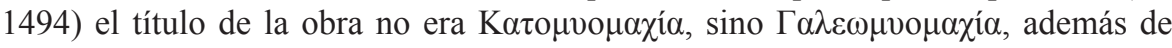
que en su "prólogo al lector" usa el término $\gamma \alpha \lambda \tilde{\eta}$ ( $\pi$ ó $\lambda \varepsilon \mu o ́ v ~ \tau \imath v \alpha ~ \gamma \alpha \lambda \tilde{\eta} \varsigma ~ \pi \lambda \alpha \sigma \alpha ́ \mu \varepsilon v o \varsigma$

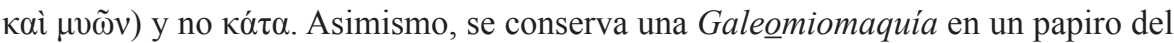

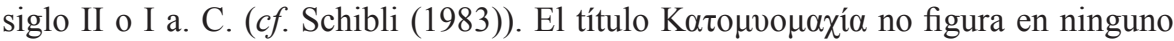
de los veinte manuscritos que conservan el poema, sino que el título que figura en cabeza del texto en los códices C (Parisinus gr. 2782 A, s. XVI), G (Parisinus suppl. gr. 608, s. XVI) y K (Parisinus suppl. gr. 1348, s. XVII) y en la hypótesis de B (Mosquensis gr. 309, s. XVI), C, F (Agen, Biblioteca Municipal, Cod. Gr. 20, s. XVI), G, H (Harleianus gr. 5664, s. XVI), L (Atenas, Universidad, Instituto Filol. Bizant. y Neohel., s. XVI), O (Baroccianus gr. 64, s. XV) y V² (V = Vindobonensis Phil. gr. 293, s. XVI; para V² $c f$. Hunger (1968: 14)) es $\Gamma \alpha \lambda \varepsilon \omega \mu v o \mu \alpha \chi i ́ \alpha$. Además, en todos los códices la palabra $\gamma \alpha \lambda \tilde{\eta}$ aparece en lugar de $\kappa \alpha ́ \tau \alpha$, que es la lectura de los dos manuscritos más antiguos, ambos del siglo XIV y anteriores a la editio princeps de Apostolio: M (Marcianus gr. 524 (ca. 1300)) y P (Vaticanus Palatinus gr. 7), en los que sólo en el verso 72 se lee $\tau \tilde{\omega} v \gamma \alpha \lambda \tilde{\omega} v$. Fue Hercher en su edición de 1873 quien restituyó la lectura de estos dos manuscritos (y también corrigió en el verso

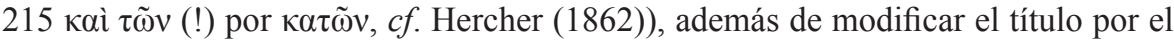

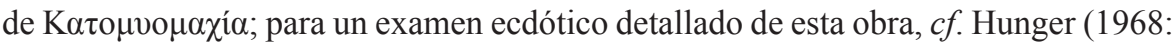


Es posible hipotetizar, entonces, que podría haber una eventual contaminación semántico-fonética entre $\gamma \alpha \lambda \tilde{\eta}$ y $\gamma \alpha \dot{\alpha} \tau(\tau) \alpha$ que daría como resultado $\kappa \alpha ́ \tau(\tau) \alpha$, por un proceso fonético de paso de la velar sonora (gamma) a la sorda (kappa) (fenómeno analogable con la ley de Grimm), aunque sería difícil explicar (si no absurdo), desde el punto de vista fonético, el cambio de la líquida de la segunda sílaba (lambda) por una (doble) dental sorda (tau). Fonéticamente, sería más sensato hipotetizar que, por influjo del latín medieval,

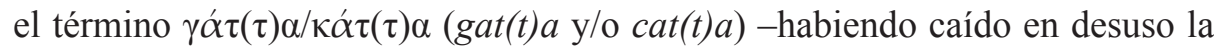

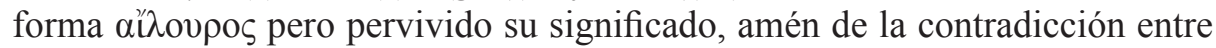
el uso griego y el latino, i.e. domesticidad vs. ferocidad-se haya infiltrado en el vocabulario bizantino vulgar, con simplificación (¿desgeminación?) de la doble tau, pudiéndose explicar tanto la ocurrencia con velar sonora (gamma) como con velar sorda (kappa) como un fenómeno de variación libre ${ }^{9}$. De ser así, y considerando que Pródromo es uno de los poetas que más y mejor ha plasmado el vocabulario popular bizantino en sus poemas, se puede conjeturar

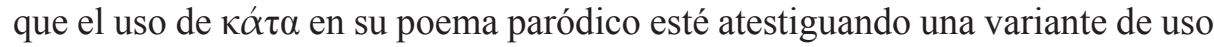
popular, extendida y quizás hasta desconocida o "estigmatizada" en los círculos cortesanos y letrados ${ }^{10}$.

Ahora bien, ¿la elección de esta variante por parte de Pródromo responde a un simple caso de variación libre o más bien a una deliberada intención comunicativa?

\section{II}

Volviendo, luego de este excursus terminológico, a la versión que presenta Pródromo de este cuento-fábula popular, cabe destacar que la acción representada en la Catomiomaquía es transmitida exclusivamente desde el

\section{3-24).}

9 "Par contre -comenta Lejeune-, la simplification des géminées, qui apparaît sporadiquement dans des inscriptions d'époque hellénistique, peut avoir un fondement phonétique: le grec moderne, s'il écrit encore des consonnes géminées, les prononce toutes comme des consonnes simples" (1947: 61).

10 Para la alternancia de registro culto/popular en el habla y en la escritura bizantinas en general, véase Horrocks (2004); para las causas que llevaron al gradual proceso de incorporación del lenguaje vulgar en la poesía bizantina, véase Signes Codoñer (2005: 43-60). 
punto de vista de los ratones. En el verso 27 uno de los personajes, Tyrokléptes ('Robaquesos'), observa que кá $\tau \alpha$ es una denominación humana (y por lo tanto

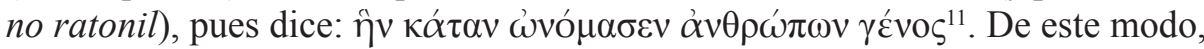
se podría estar llamando la atención (desde el punto de vista ratonil) sobre el significante ['ka ta] ${ }^{12}$, por lo que sería posible inferir que habría una sutil pero decisiva diferencia entre la conceptualización humana y la de los ratones,

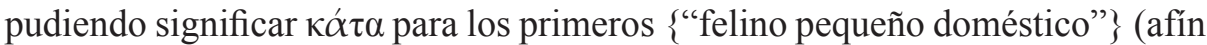
por sus componentes semánticos a $\alpha\left(\iota^{\prime} \lambda o v \rho o \varsigma\right)$, mientras que para los segundos significaría todo lo contrario \{"felino grande salvaje"\}. De ser así, desde el punto de vista de los ratones (que es con el que Pródromo quiere que se identifique(n) el/los oyente/lector/es) el gato siempre es asesino y nunca domesticable. Tal es así, que la coincidencia entre el punto de vista humano y el punto de vista ratonil empieza y termina ahí. Comparten un significante. Nada más. Ká $\alpha \alpha$ no es más que eso: una denominación. De hecho, los ratones se refieren al gato de muchas otras maneras y todas ellas con connotaciones de peligro, rapiña y muerte. Esto se puede ilustrar con los atributos con que estos califican al gato y sus acciones.

En efecto, lo/la llaman: rapaz urdidor de engaños ( $\tau \tilde{\eta} \varsigma$ $\alpha \rho \pi \alpha \gamma o \varsigma \tau \tilde{\eta} \varsigma$

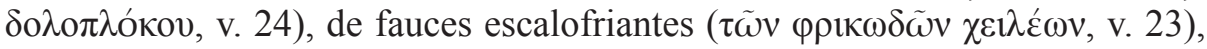

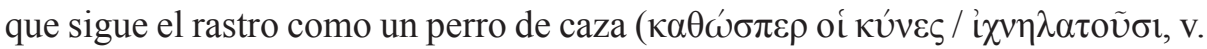

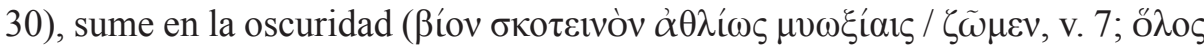

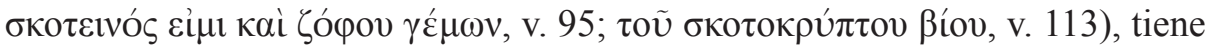

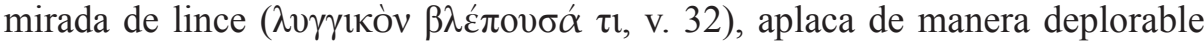

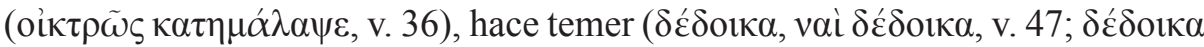

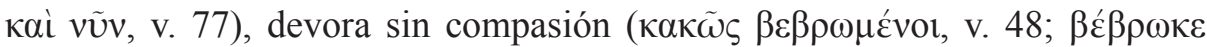

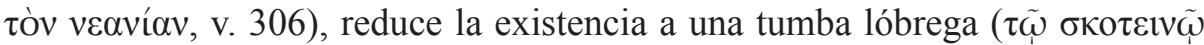

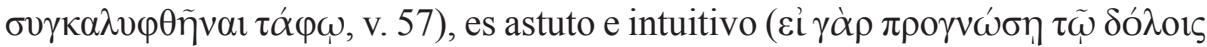

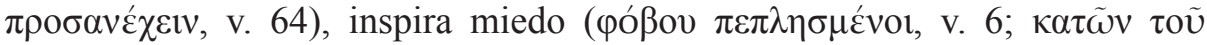

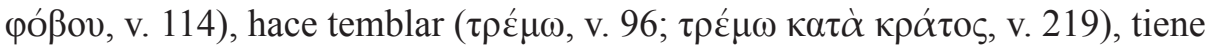

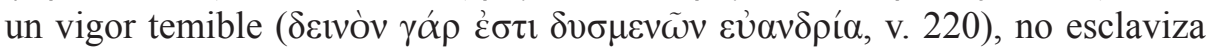

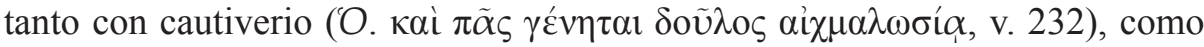

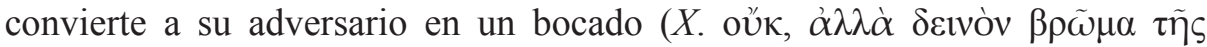
$\dot{\varepsilon} v \alpha v \tau i ́ \alpha \varsigma$, v. 233), es voraz ( $\beta \rho \tilde{\omega} \mu \alpha \tau \tilde{\eta} \varsigma \dot{\alpha} \delta \eta \varphi \alpha ́ \alpha o v$, v. 237), hace gemir ( $\pi \alpha \tilde{v} \sigma \alpha 1$

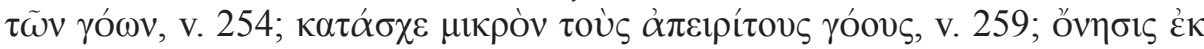

11 En este trabajo se sigue el texto establecido por H. Hunger (1968).

12 Se usa aquí la notación fonética-fonológica para transcribir el significante puro. 


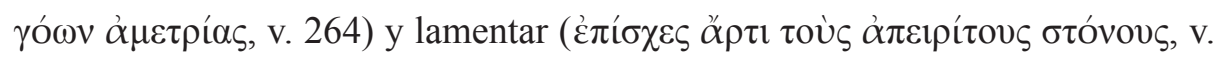

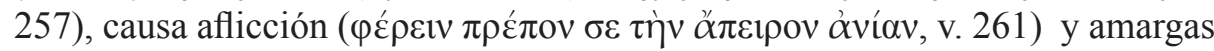

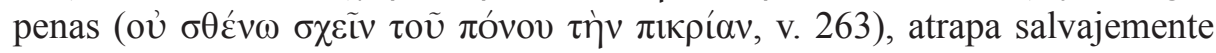

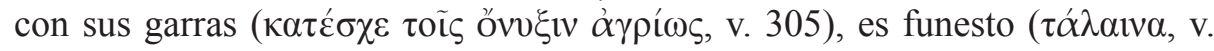

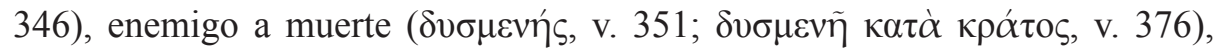

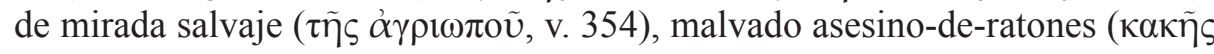

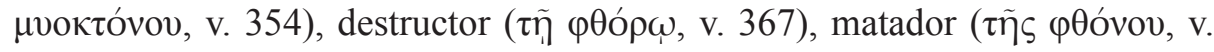

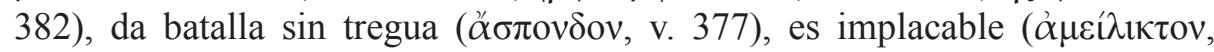

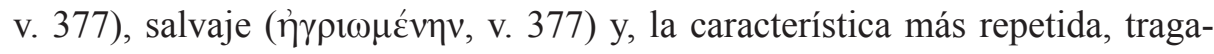

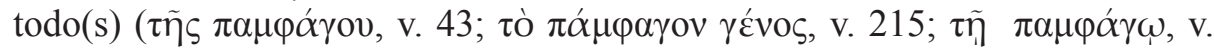

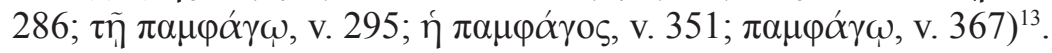

Se podría pensar, pues, que desde el punto de vista ratonil no es

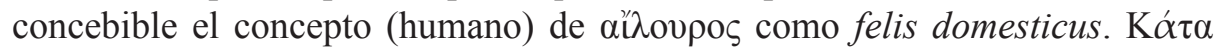
sería, entonces, para los ratones un término cargado de connotaciones exclusivamente negativas, atribuciones semánticas que "cortocircuitarían" y desautomatizarían la percepción cotidiana del oyente/lector (humano), quien tiene conceptualizado al gato de modo opuesto (doméstico y manso, no salvaje y asesino), disrupción semiótica que habilita un proceso de decodificación que

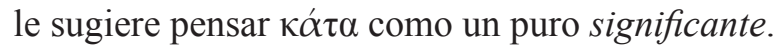

\section{III}

A principios del siglo pasado, como es sabido, un grupo de críticos y teóricos rusos, luego conocido como formalistas rusos, intentó pensar "científicamente" la literatura. En una primera aproximación pensaron que lo específico de toda obra literaria se reducía a una serie de procedimientos

13 Estos sentimientos y sensaciones evocados por el personaje del gato habrían encontrado una íntima aceptación por parte del público bizantino, ya que, como observa Runciman, "acosados por el temor y la inseguridad, los bizantinos no podían dejar de ser recelosos y de tener unos nervios fácilmente excitables y propicios a la irritación y al pánico" (Runciman (1942: 202)), del mismo modo que el género paródico-trágico de esta obra prodrómica les habría resultado muy cercano a su sensibilidad estética, pues "el espíritu bizantino era agrio; su humor encontraba su expresión en la burla y en el sarcasmo. En efecto, la vida parecía una burla" (op. cit.: 203), así como su tono sombrío: "este gran imperio, el último hogar de la civilización en un mundo sombrío y borrascoso, estaba continuamente tambaleándose entre los bárbaros, y sólo se recobraba para encontrarse con un nuevo ataque" (op. cit.: 203). 
que llamaban la atención sobre la obra en sí misma, confiriéndole el status de objeto estético. Uno de esos procedimientos, quizás el más reconocido, fue el que puso en evidencia V. Shklovsky: el procedimiento de 'extrañamiento' (ostranenie). Según este procedimiento artístico-literario, la obra llama la atención sobre sí misma en tanto puro significante con el propósito de aumentar la duración de la percepción ${ }^{14}$. Semióticamente, se hace crecer de manera desmesurada el significante en detrimento del significado, siendo la actividad perceptiva del espectador-lector un elemento clave para el funcionamiento de este procedimiento.

Con esto en mente, y ya intentada la clarificación léxica del término

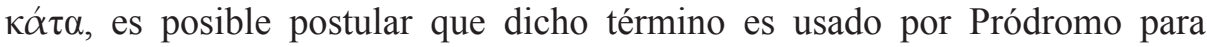
activar el 'efecto de extrañamiento’ que requeriría del oyente/lector un esfuerzo interpretativo para colaborar en la asignación de un designado a este término extrañificado. Ese requerimiento de colaboración evocaría un doble lector y una doble situación de lectura ${ }^{15}$.

Por un lado, remitiría al lector-oyente que recibe la obra en el interior de la corte Comnena, escuchando en voz alta el recitado del texto ${ }^{16}$; en este caso, el

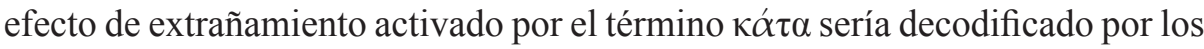

14 V. Shklovsky enfatiza el elemento procedimental y perceptivo en su definición del 'efecto de extrañamiento': "nosotros llamaremos objetos estéticos (...) a los objetos creados mediante procedimientos particulares, cuya finalidad es la de asegurar para estos objetos una percepción estética (...) los procedimientos del arte son el de la singularización de los objetos y el que consiste en oscurecer la forma, en aumentar la dificultad y la duración de la percepción" (Shklovsky (1917: 57)); de aquí deriva su célebre caracterización de tal procedimiento como desautomatización de la percepción: "para dar sensación de vida, para sentir los objetos, para percibir que la piedra es piedra, existe eso que se llama arte. La finalidad del arte es dar una sensación del objeto como visión, y no como reconocimiento" (op. cit.: 60).

15 Para un panorama detallado de las diversas modalidades de lectura practicadas en Bizancio, puede verse el erudito estudio de G. Cavallo (2004).

16 En su introducción a la Catomiomaquía, García Romero observa que esta obra era un dramátion "no para la representación, sino para la lectura" (2003: 5); "contamos con un serie de informaciones -señala Signes Codoñer- que nos indican que buena parte de la literatura bizantina estaba destinada a ser recitada, bien en audiciones más o menos privadas de círculos de la corte, bien en ceremoniales públicos" (Signes Codoñer (2005: 48)). 
miembros de la corte y sus circunstantes como una referencia al múltiple peligro evocado por el enemigo exterior ${ }^{17}$ que acecha y amenaza constantemente al Estado Bizantino desde fines del siglo XI y durante todo el siglo XII y sume la existencia del hombre (y de la mujer) bizantino en un constante estado de alerta: el asedio de los turcos selyúcidas, las pretensiones normandas, la presión de los Cruzados y el creciente establecimiento de comerciantes venecianos en territorio bizantino (ipeligros que todo lo devoran, como el gato en el poema!) ${ }^{18}$.

Por otro lado, remitiría Pródromo a otros pares letrados, profesore ${ }^{19} \mathrm{o}$ cofrades $^{20}$, quienes leyendo (individualmente) el texto en voz baja o en silencio ( ien la oscuridad, como los ratones!) ${ }^{21}$, verían simbolizadas con el término

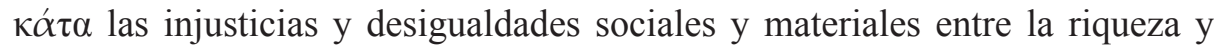
abundancia que ostentan los estratos superiores y la pobreza y carencia que padecen los sectores subordinados, en consonancia con los temas recurrentes de la pobreza y el hambre que sufren aquellos miembros de la sociedad bizantina dedicados al trabajo intelectual (profesores, poetas, gramáticos, monjes), temas presentes en los poemas "ptokhoprodrómicos", especialmente en el tercero ${ }^{22}$, donde se satiriza contra la actitud voraz de los abades que matan de hambre ${ }^{23}$

17 Ajenidad que, como posible latinismo, connotaría la palabra кó $\alpha \alpha$ para una corte que hacía de la lengua griega "pura" vehículo de la identidad nacional, de la alta cultura y de la fe ortodoxa ( $c f$. Horrocks (2004: 461-465)).

18 Estos peligros y amenazas, corolario del terrible desastre de Manzikert y de la pérdida de Bari en 1071, desembocarán en la derrota de Miriocéfalo en 1176, preludio de la crisis financiera y política de 1204 que pondrá en jaque al Imperio Bizantino; cf. Runciman (1942), Baynes (1951), Maier (1982), Romero (2006), Kaplan (2007).

19 Para la situación de los profesores en la sociedad bizantina, véase Browning (1994).

20 Para la problemática relación del monje con la lectura solitaria, véase Schreiner (2004).

21 La lectura murmurada o silenciosa era en Bizancio una práctica marginal, ligada desde la Antigüedad con la lectura individual de fábulas o relatos: "en el mundo antiguo, en definitiva, el hablar a media voz, el susurro o el silencio se asocian a la lectura solitaria, íntima y a lo fantástico, mágico, novelesco" (Cavallo (2002: 68)).

22 Según Egea (2001), quién sigue las ediciones de Hesseling - Pernot (1910: III) y de Eideneier (1991: IV).

23 Ténganse en cuenta todos los nombres de los ratones mencionados en el

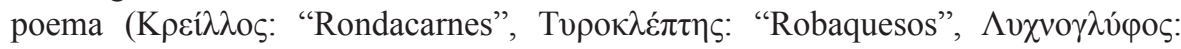

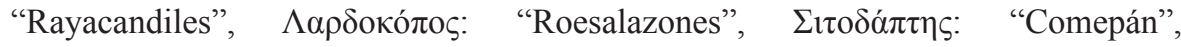

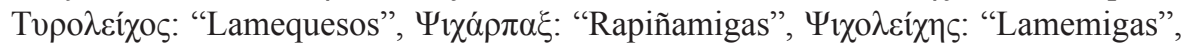


a sus monjes.

Esta (doble) interpelación al(os) oyente(s)/lector(es) se podría ver manifiestamente señalada en el cuerpo textual con estas palabras del mensajero

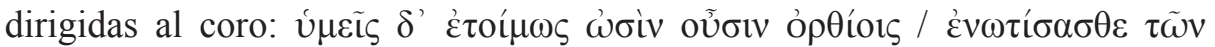

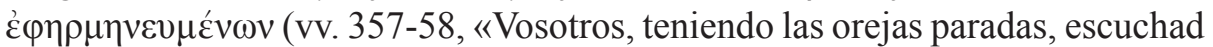
[¡ahora!] con atención los hechos explicados»), quien(es) estaría(n) dividido(s) en dos grupos ${ }^{24}$ :

(a) los miembros de la corte, aludidos como cuerpo de soldados aunados en la preparación de la batalla con su interés eclesiástico-estatal: $\kappa \alpha i$

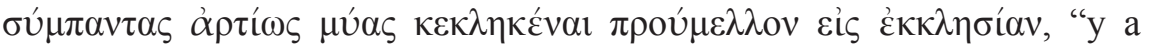
todos los ratones tenía en seguida la intención de convocar en asamblea",

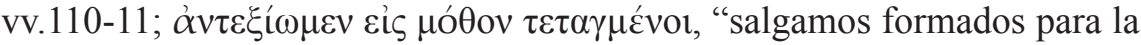

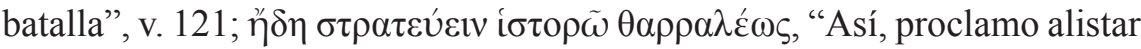

К

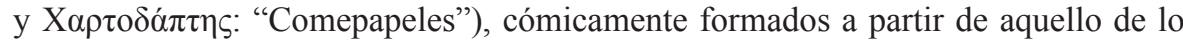
que se alimentan (aunque una mención especial requeriría Khartodáptes, linaje del que se enorgullece descender el rey Kreíllos, que en tanto "tragapapeles" podría ser una máscara del propio Pródromo como "rata de biblioteca", es decir, como erudito letrado), por lo que con estos podría estar sugerido el tópico de la pobreza y el hambre, verdadero Leitmotiv de sus poemas "mendicantes"; un estudio detallado de la figura del pobre en la sociedad bizantina puede verse en Patlagean (1994), quien distingue dos categorías principales de pobres: por un lado, el pénes, que, si bien realiza alguna actividad, esta resulta insuficiente para garantizar su subsistencia; por otro, el potkhós, quien, reducido a la postración y a la inacción, espera todo de los demás.

24 Según Iser, todo texto literario tiene algo de 'performativo', pues no expone ni describe sino que produce reacciones causadas por la realidad, dado que "no poseen objetos que le correspondan exactamente en el «mundo de la vida», sino que obtiene sus objetos a partir de elementos que se encuentran en ese mundo" (1987: 135). Por ello, configura y proyecta el esbozo de una estructura objetiva mediante la que se apela al lector, marcando las posibilidades de decodificación de cada texto, al garantizar un "espacio de juego de posibilidades de actualización" (op. cit.: 134), ya que su sentido no radica en el mundo sino en el proceso de lectura que posibilita; sentido, sin embargo, que acusa siempre cierta indeterminación a causa de la total falta de adecuación tanto al mundo real como a las experiencias del lector: "ahí radica la especificidad del texto literario. Se caracteriza por una típica oscilación entre el mundo de los objetos reales y el mundo de la experiencia del lector" (op. cit.: 137). 


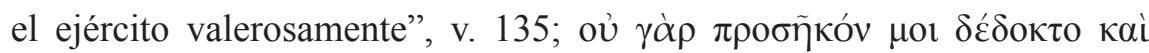

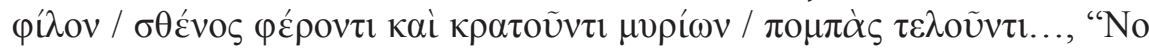
me parecía, pues, decoroso, que teniendo fuerza y gobernando sobre miles,

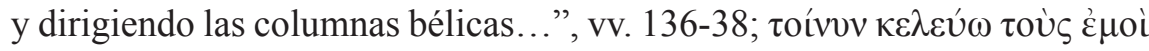

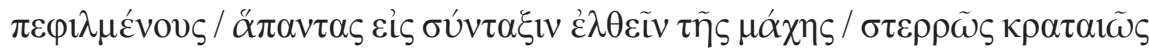

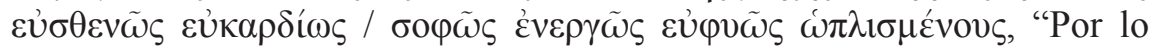
tanto, ordeno a todos los que me son queridos acudir a las formaciones de la batalla, equipados de robustez, de poder, de vigor, de buen ánimo, de sabiduría, de energía y de talento natural", vv. 173-76.

(b) otros "colegas" letrados o cofrades, aludidos como cuerpo de hermanos unidos en su interés grupal y social: $\sigma 0 ́ v \alpha \mu \varepsilon$, "hermano consanguíneo",

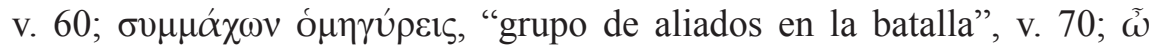

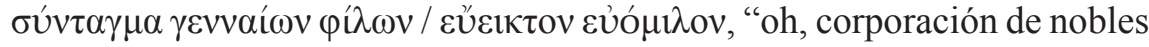

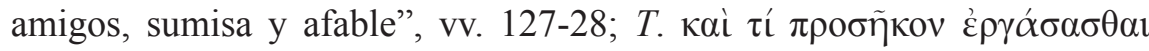

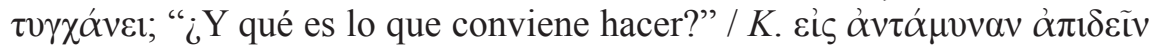
$\tau \tilde{\eta} \varsigma \pi \alpha \mu \varphi \alpha ́ \gamma \gamma o v$, "Mirar bien para defendernos del tragatodo(s)", vv. 42-43.

Nótese, asimismo, que hay personajes que relatan lo ocurrido, como el ángelos en los vv. 283-306 y 356-378. Relato, rhêsis, de los mensajeros que,

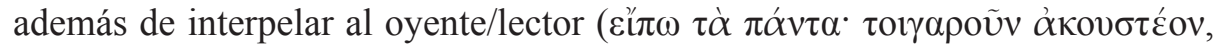
"habré de contarlo todo; por lo tanto, tiene que ser escuchado", v. 283) ${ }^{25}$, instala en el texto la figura del 'Autor Modelo', representante en el cuerpo textual del 'Autor Empírico' y guía e instrucción para el lector del modo y de la situación de lectura que requiere para su adecuada decodificación ${ }^{26}$.

25 Considerando el contexto de uso del adjetivo verbal derivado del verbo akoúo, utilizado en el verso 283 , y los términos formados a partir de su raíz (*ak-) y su

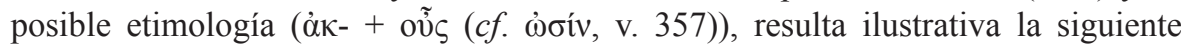

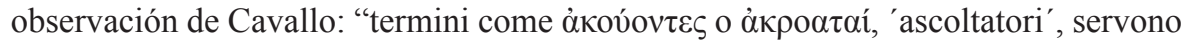
sovente ad indicare anche i 'lettori'" (2004: 573), dado el rol fundamental de que habría gozado la voz en la modalidad de lectura más habitual (la sonora) practicada en Bizancio (por ejemplo, la epistolografía, "quizás el género literario más cultivado y apreciado en la cultura bizantina" (Cavallo (2002: 63)).

26 Para la idea de 'Autor Modelo' consultar Eco (2000: 87-95); ver nota siguiente. 
De este modo, la Catomiomaquía estaría propiciando dos situaciones de lectura, dos 'Lectores Modelo' ${ }^{27}$ que el poema requeriría para hacer circular su velado mensaje paródico-político-crítico ${ }^{28}$, contra un doble enemigo ${ }^{29}$, asesino y feroz en ambos casos, al que no es posible "domesticar".

A modo de conclusión, pues, es posible pensar que para ambos lectores el enemigo no puede ser concebido de otra manera, del mismo modo que a los

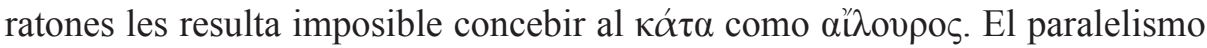
entre el punto de vista del ratón y el punto de vista del ciudadano bizantino/hombre de letras instalaría, mediante un efectivo procedimiento de 'extrañamiento', la visión, la idea, la concepción de que el enemigo es indomable, temible y cruelmente implacable. No hay alternativa. No hay posibilidad de diálogo. O bien la muerte propia o bien la muerte del extraño/otro. Esta valoración del enemigo 'no cristiano' -uno(s) por profesar otra religión (selyúcidas) o bien por profesar la misma pero con otro credo (Cruzados), otro(s) por transgredir y abusar de los principios cristianos garantes de la cohesión social (como, por ejemplo, el del amor al prójimo o el de la igualdad en el sufrimiento (emperadores y abades))- como fiero y salvaje constituiría uno de los pilares ético-político-religiosos de la cultura y de la sociedad bizantina.

27 La noción de 'Lector Modelo' fue propuesta por U. Eco para designar el tipo de lector que cada texto en particular postula como condición indispensable de su actualización, posicionándolo en el tejido textual y constituyéndolo a través de huellas textuales, cuya función sería la de colaborar en la construcción del sentido que todo texto (sobre todo los artístico-literarios) requeriría para funcionar como tal; la "necesariedad" de este principio pragmático de cooperación interpretativa reside, según Eco, en que "un texto es un mecanismo perezoso (o económico) que vive de la plusvalía de sentido que el destinatario introduce en él (...) Un texto quiere que alguien lo ayude a funcionar" (2000: 76); un examen más detallado de esta noción puede verse en Eco (2000: 73-95).

28 Intencionalidad crítica del poeta contra los superiores tanto del Estado como del alto clero, quizás favorecida por el hecho de que durante la época de los Comnenos en las instituciones eclesiásticas "la anarquía tendía con frecuencia a relajar su disciplina. Los monasterios, por ejemplo, necesitaban una vigilancia constante" (Runciman (1942: 119)).

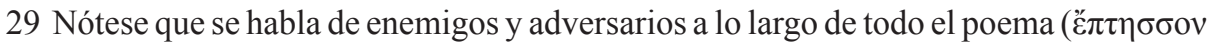

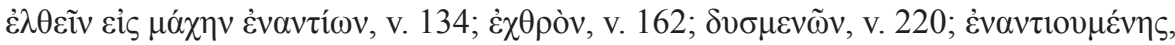

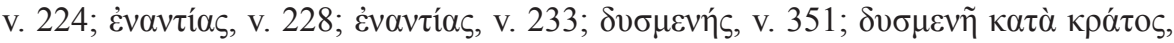
v. 376). 
Pero también, esta valoración negativa haría oír la voz, la queja silenciosa y silenciada del hombre de letras (y junto con él de aquellos miembros del cuerpo social) que ve(n) con injusticia e impotencia cómo los poseedores del poder político y religioso mantienen en la "oscuridad" y en el hambre a quienes los celebran y legitiman en su inexpugnable condición de poder $^{30}$.

30 Un eco de la voz de este cuerpo de los subyugados puede verse en lo que dice el coro en el verso 218 a propósito de su posibilidad de entablar con éxito la batalla contra

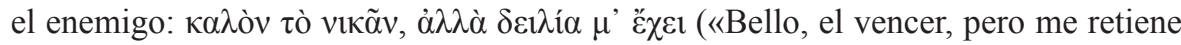
la cobardía»). Esta suerte de $\gamma \nu \omega ́ \mu \eta$ negativa de efecto paralizante sería el correlato del planteamiento hecho entre el rey Kreillos y Tyrokléptes en el prólogo dialogado

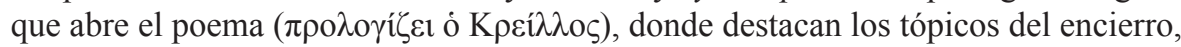
la oscuridad, el miedo, la impotencia y la existencia miserable (cf. Catomiomaquía, vv. 1-32). 
DANIEL GUTIÉRREZ: Tragatodo(s): el cuento del gato y los ratones...

\section{REFERENCIAS BIBLIOGRÁFICAS}

APOSTOLIUS, ARISTOBULUS (ca. 1494). Г $\alpha \lambda \varepsilon \omega \mu v o \mu \alpha \chi i \alpha$, editio princeps, Bibliotheca Augustana Disponible en: https://www.hsaugsburg.de/ hrasch/graeca/Chronologia/ S_post12/Prodromo/pro_kat0.htm BAILLY, A. (1963). Dictionnaire grec-français. Paris: Hachette.

BAYNES, N. H. (1951). El Imperio Bizantino. México: FCE.

BEEKES, R. (2009). Etymological Dictionary of Greek. Leiden and Boston.

BROWNING, R. (1994). "El profesor", en G. Cavallo (comp.), El hombre bizantino. Madrid: Alianza, 125-152.

CARACAUSI, G. (1990). Lessico greco della Sicilia e dell'Italia meridionale (secoli X-XIV). Palermo: Centro di Studi Filologici e Linguistici Siciliani.

CAVALLO, G. (comp.) (1994). El hombre bizantino. Madrid: Alianza.

-, (2002). "Entre voz y silencio: de la lectura antigua a la lectura medieval", Estudios Clásicos, 44, 63-71.

-, (2004). "Le pratiche di lettura", en La cultura bizantina, vol. I, Lo spazio letterario del Medioevo, 3: le cultura circostanti. Roma: Salerno, 569604.

CHANTRAINE, P. (1968). Dictionnaire étymologique de la langue grecque. Histoire des mots. Paris: Klincksieck.

DIMITRAKOS, D. (1951). Méga lexicon ólis tis ellinikís glóssis. Athinai: Domi.

DU CANGE, CH. (1958). Glossarium ad scriptores mediae et infimae graecitatis. Lugduni: Anissonius-Posuel-Rigaud, $1668=$ Graz, 1958.

ECO, U. (2000). Lector in fabula. La cooperación interpretativa en el texto narrativo. Barcelona: Lumen.

EGEA, J. M. (2001). Versos del gramático señor Teodoro Pródromos el Pobre o Poemas Ptocoprodrómicos. Granada: Centro de Estudios Bizantinos, Neogriegos y Chipriotas.

EIDENEIER, H. (1991). Ptochoprodromos: Einfürung, kritische Ausgabe, deutsche Uebersetzung, Glosar. Colonia: Romiosini Verlag (Neograeca Medii Aevi).

ERNOUT, A. - Meillet, A. (1959). Dictionnaire Etymologique de la Langue Latine. Paris: Klincksieck.

FLACELIÈRE, R. (1959). La vida cotidiana en Grecia en el siglo de Pericles. Buenos Aires: Hachette.

GARCÍA ROMERO, F. A. (2003). Teodoro Pródromo: La Catomiomaquía. Edición, traducción, introducción y notas. Jerez de la Frontera: Real Academia de San Dionisio de Ciencias, Artes y Letras y Centro de Estudios Históricos Jerezanos. 
HERCHER, R. (1862). "Ptockoprodromika", Rheinisches Museum, 17, 411 SS.

-, (1873). Theodori Prodromi Catomyomachia ex recensione Rudolfi Hercher. Lepizig: Bibliotheca Teubneriana.

HESSELING, D. C. - Pernot, H. (1910). Poémes Prodromiques en grec vulgaire. Amsterdam.

HORROCKS, G. (2004). "Lingua alta e lingua popolare", en G. Cavallo (ed.), La cultura bizantina, vol. I, Lo spazio letterario del Medioevo, 3: le cultura circostanti. Roma: Salerno, 457-490.

HUNGER, H. (1968). Der byzantinische Katz-Maüse-Krieg. Theodoros Prodromos, Katomyomachia, Einleitung, Text und Übersetzung. Graz-Viena-Colonia: Verlag Hermann Böhlaus Nachtf. (Byzantina Vindobonensia).

-, (1978), Die hochsprachliche profane Literatur der Bizantiner, Munich, Beck.

ISER, W. (1987). "La estructura apelativa de los textos", en En busca del texto. Teoría de la recepción literaria, Dieter Rall (comp.). México: UNAM.

KAPLAN, M. (2007). Byzance. Paris: Les Belles Lettres.

KAZHDAN, A. (ed.) (1984). Studies on Byzantine Literature of the Eleventh and Twelfth Centuries. Cambridge: Cambridge University Press.

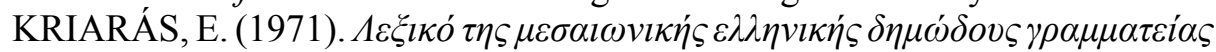
1100-1669. Tesaloniki.

LAMPE, W. (1961). A patristic greek lexicon. Oxford: Clarendon.

LEJEUNE, M. (1947). Traité de phonétique grecque. Paris: Klincksieck.

LEMERLE, P. (1971). Le premier humanisme byzantine. Paris: PUF.

LEWIS, CH. - SHORT, CH. [=L\&S] (1962). Latin Dictionary. Oxford.

LIDDELL, H. - SCOTT, R. - Jones, H. [=LSJ] (1996). A Greek-English Lexicon. Oxford.

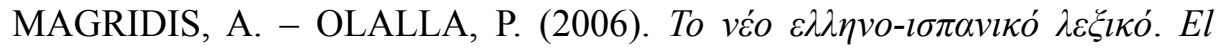
nuevo diccionario griego-español. Atenas: Texto.

MAIER, F. G. (1982). Bizancio. Madrid: Siglo XXI.

NIERMEYER, J. (1954-1958). Mediae Latinitatis lexicon minus. Leiden: Brill, Fasciculi 1-6.

OXFORD LATIN DICTIONARY [=OLD] (1968-1982). Oxford: Clarendon Press.

PASSOW, F. (1993). Handwörterbuch der griechischen Sprache, Neue bearbeitet und zeitgemä $\beta$ umgestaltet von Val. Fr. Rost, Friedrich Palm Otto Kreussler, Karl Keil und Ferd. Peter. Wissenschaftliche Buchgesellschaft: Darmstadt.

PATLAGEAN, E. (1994). "El pobre", en G. Cavallo (comp.), El hombre bizantino. Madrid: Alianza, 27-59. 
PERRY, B. E. (1952). Aesiopica. Chicago.

ROMERO, J. L. (2006). La Edad Media. Buenos Aires: FCE.

RUNCIMAN, S. (1942). La civilización bizantina. Madrid: Pegaso.

SCHIBLI, H. S. (1983). "Fragments of a Weasel and Mouse War", Zeitschrift für Papyrologie und Epigraphic, 53, 1-26.

SCHREINER, P. (2004). "«Il libro è la mia cella». Le letture monastiche", en G. Cavallo (ed.), La cultura bizantina, vol. I, Lo spazio letterario del Medioevo, 3: le cultura circostanti. Roma: Salerno, 605-631.

SHKLOVSKY, V. (1917). "El arte como artificio", en T. Todorov (ed.), Teoría de la literatura de los formalistas rusos. Buenos Aires: Siglo XXI, 2004, 55-70.

SIGNES CODOÑER, J. (2005). "Poesía clasicista bizantina en los siglos X-XII: entre tradición e innovación”, en V. Valcárcel Martínez - C. Pérez González (eds.), Poesía medieval (Historia literaria y transmisión de textos). Madrid: Fundación Instituto Castellano y Leones de la Lengua, 19-66.

SOPHOCLÉS, E. (1992). Greek lexicon of the Roman and Byzantine periods. Hildesheim-Zürich-New York: Georg Olms Verlag.

TRAPP, E. (2001). Lexicon zur byzantinistischen Gräzität, besonders des 9.12. Jahrhunderts. Wien: Österreichischen Akademie der Wissenschaften, Band A-K. 